Conclusions Inclusion of Quebec's risk exposure data in the national HIV dataset is significant; the national dataset becomes more complete and the proportion of cases with unknown exposure category is reduced. This analysis demonstrates that inclusion of exposure category data, from the provincial HIV surveillance system of Quebec's INSPQ can alter the exposure category breakdowns at the national level, thereby offering a more accurate picture of HIV diagnoses in Canada.

\section{P1-S4.07 THE USE OF THE ARIZONA DEPARTMENT OF HEALTH SERVICES SURVEILLANCE DATABASE TO IDENTIFY DISCORDANT SYPHILIS TREPONEMAL LABORATORY RESULTS, ARIZONA}

doi:10.1136/sextrans-2011-050108.151

M Winscott, A Betancourt, R Ereth. Arizona Department of Health Services, Phoenix, USA

Background In March of 2010 a large commercial lab performing STD testing in Arizona implemented the reverse algorithm for syphilis testing with the use of the syphilis treponemal enzyme immunoassay (EIA).

Methods Per the Arizona Department of Health Services (ADHS) Sexually Transmitted Disease Control Program protocol, manual review of positive syphilis lab results is performed by one epidemiologist. During May through October 2010, this epidemiologist recorded negative syphilis EIA results identified among reported syphilis cases with a prior history of positive treponemal tests (TP-PA, FTA-ABS).

Results From 6 May 2010 to 29 October 201078 syphilis EIA tests were reported as negative in individuals with a previously positive treponemal test. Fifty of these tests were completed among males and 28 among females. This discrepancy was brought to the attention of the reporting lab in May and again in October 2010. In November, a coordinated investigation between the reporting laboratory, the ADHS public health lab, and the ADHS Sexually Transmitted Disease Control Program (ADHS STDCP) was undertaken. This investigation resulted in the identification and correction in a specimen transfer procedure at the local laboratory level. All providers of patients with possible incorrect lab results completed during this time period were notified of the problem and advised to retest their patients, if indicated. The ADHS STDCP continues to monitor syphilis lab results for this particular error and has not identified any further discordant results since the corrections were implemented in November 2010.

Conclusions Discrepancies in syphilis EIA results have been reported from other regions. The detailed manual review of all reported syphilis lab results by an epidemiologist at the ADHS STDCP aided in the detection of this particular issue. Upon implementation of electronic lab reporting, this program and others, should continue routine, manual quality assurance checks of syphilis laboratory results. Such practices are imperative to ensure that these errors do not go undetected and that syphilis patients do not go undiagnosed.

\section{P1-S4.08 POPULATION SIZE ESTIMATES FOR MEN WHO HAVE SEX WITH MEN IN GUATEMALA CITY USING TIME LOCATION SAMPLING AND RESPONDENT DRIVEN SAMPLING}

doi:10.1136/sextrans-2011-050108.152

${ }^{1} \mathrm{G}$ Paz-Bailey, ${ }^{2} \mathrm{~B}$ Alvarez, ${ }^{2} \mathrm{~W}$ Miller, ${ }^{3} \mathrm{~B}$ Sabrina, ${ }^{4} \mathrm{C}$ Barrington, ${ }^{5} \mathrm{~A}$ Kim, ${ }^{2} \mathrm{~S}$ Morales, ${ }^{5} \mathrm{~S}$ Chen. ${ }^{1}$ Tephinet Inc, Guatemala, Guatemala; ${ }^{2}$ Del Valle University of Guatemala, Guatemala, Guatemala; ${ }^{3}$ Tephinet Inc, Guatemala; ${ }^{4}$ University of North Carolina, USA; ${ }^{5}$ Centers for Disease Control and Prevention, USA

Background Men who have sex with men (MSM) are highly vulnerable to HIV infection, but this population can be particularly difficult to reach in Central America due to stigma and violence. Knowing the size of populations at high risk for HIV and sexually transmitted infections (STI) is critical for informing prevention, care, and treatment programs. Simple approaches are needed to provide baseline estimates of the population size of MSM in Guatemala to advocate for appropriate resource allocation and programming. We compared population size estimates of MSM and transgender persons using capture-recapture linked to two differentsurveys that were developed concurrently using different sampling methodologies.

Methods Capture recapture methods for estimating population size were integrated into two probability-based surveys using respondent driven sampling (RDS) and time-location sampling (TLS); conducted simultaneously among MSM in Guatemala City from October to December 2010. Key chains were used as uUnique objects in the form ofand study key chains were distributed approximately 1-month prior to the surveys in venues known to be frequented by MSM. Duplicate participation was avoided by using the same team to distribute the key chains and administering a set of questions before handing the object. Participants in the RDS and TLS surveys were asked, as part of the study interview, whether they had received the key chain. We assigned the number of key chains distributed in venues as the first capture and the proportion of participants that reported receiving the key chain in the RDS or TLS survey as the second capture. By applying standardised formulas for estimating population size using capture-recapture methods, we estimated the number of MSM in Guatemala City and 95\% CIs around this estimate, adjusted for RDS and TLS sampling design.

Results A total of 2128 key chains were distributed in the first capture. Of the 501 MSM interviewed in the RDS survey, 200 (RDS adjusted, $23.4 \%$ ) had received the key chain. Thus the RDS adjusted estimated population size of MSM in Guatemala City was 9,190 (95\% CI 7765 to 10616). Of the 504 MSM interviewed in the TLS survey, 193 (TLS adjusted, 32.1\%) had received the key chain. The TLS adjusted estimated number of MSM was 6620 (95\% CI 5813 to 7427 ).

Conclusions Estimates of MSM population size using the TLS survey resulted in a significantly lower estimate than that obtained through RDS survey, probably due to the fact that TLS targets MSM who attend venues. The estimate obtained through RDS may better reflect the size of the larger MSM population in Guatemala City, since this itRDS reaches MSM attending and not attending publicmeeting venues. Integrating capture-recapture methods in probability-based surveys among MSM is a simple and fast approach for providing the population sizes needed to inform and evaluate programs for MSM in Guatemala.

\section{P1-S4.09 VALIDATION OF THE HEDIS MEASURE OF SEXUAL ACTIVITY IN ADOLESCENTS}

doi:10.1136/sextrans-2011-050108.153

S Wiehe, J D Fortenberry, M Rosenman, W Tu. School of Medicine, Indiana University, Indianapolis, USA

Background The proportion of sexually active young women who are screened annually for chlamydia is a Health Plan Employer Data and Information Set (HEDIS) performance measure. The accuracy of this measure, however, depends critically on how the denominator is calculated (ie, how many sexually active young women for whom screening is indicated). Few studies have assessed whether the measure is valid. Validating is difficult because sexual activity may not be routinely captured in the chart or other administrative data sources. Our objective was to determine the sensitivity and specificity of the HEDIS measure of sexual activity, by comparing it to a selfreported measure, and assess whether these vary by age, age of first sex, and prior sexually transmitted infection (STI) or pregnancy. 